\title{
SIGNIFICANCE OF IMAGE-CHARGE FIELDS ON THE WRITE PERFORMANCE
} OF A POLE-KEEPER HEAD.

Martin F. Beusekamp and Jan H. Fluitman

Abstract - This paper presents a method for the computation of the magnetization and magnetic-field distributions of one transition in a perpendicular recording layer. It is used in a thick single-pole head keeper layer configuration to show that inage-charge effects are significant and have a geometrydependent impact on both height and gradient of the transition.

\section{INTROOUCTION}

In the discussion concerning the merits of perpendicular recording compared to longitudinal recording, Iwasaki et al. stated that the interaction between the magnetized recording layer and the main pole gives rise to "steep field distributions" [1], while on the other hand Mallinson et al. stated that the field gradients of pole-keeper heads are "virtually identical" to those of ring heads [2]. In this paper, the exact shape of a magnetization transition written by a pole-keeper head of given geometry is computed and the accompagnying fields are analysed.

The geometry of the investigated recording system is given in fig. 1, which shows the right-hand edge of a thick single pole head (e.g. Ohtsubo [3]) opposite a perpendicular recording layer with thickness $t$ and separated from this main pole by an air gap $\mathrm{g}$. The recording layer is backed by a keeper layer with an assumed $\mu=\infty$ and has an initial magnetization $M_{0}$ in the direction as drawn. The recording layer material is assumed to have a rectangular (intrinsic) hysteresis loop with $M_{s}>H_{C}$ (fig. 2). The head can induce a field opposite to $M_{0}$, thereby writing one transition in the region near the edge of the head.

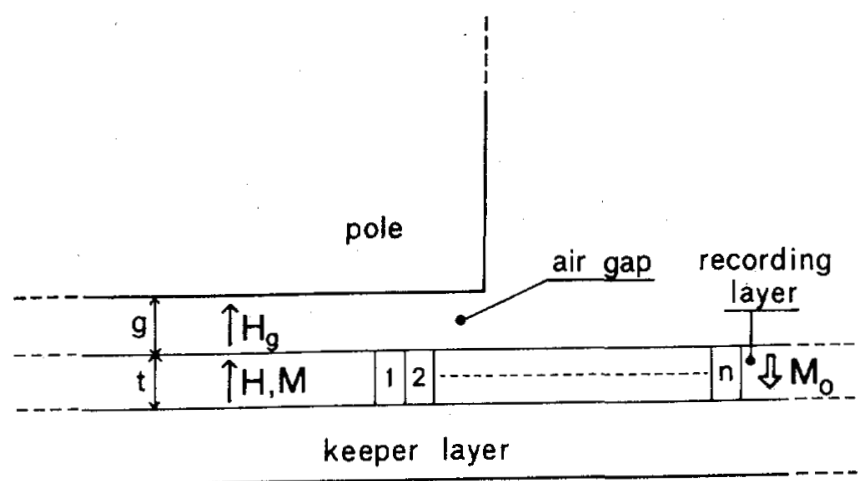

Fig. 1. Geometry of pole-keeper head and recording layer.

\section{RECORDING PROCESS IN THE DEEP GAP REGION}

In the region inside the head and far from the edge (fig. 1), Maxwell's laws can be written as:

$$
\begin{aligned}
& g \cdot H_{g}+t \cdot H=n I \\
& H_{g}=H+M
\end{aligned}
$$

From these equations it is clear that, without a magnetomotive force driving the head $(\mathrm{nI}=0), \mathrm{H}_{\mathrm{g}}$ will not equal zero and will be opposite to the self-demagnetizing field $\mathrm{H}$. Eliminating $\mathrm{H}_{\mathrm{g}}$ from (I) and (2) will give:

$$
H=\frac{n I}{(g+t)}-M+\frac{M t}{(g+t)}
$$

Manuscript received February 27, 1985.

The authors are with Twente University of Technology, P.O. Box 217, 7500 AE Enschede, The Netherlands.
The first term in (3) is the head field as it would occur in the absence of a recording layer [2], the second term is the self-demagnetizing field in the layer $(H=-M)$, while the third term must be ascribed to the image-charge field, caused by the reflection of magnetic charge of the recording layer in the pole piece and keeper layer. From (3), the significance of the image-charge field is obvious, especially when $\mathrm{g} / \mathrm{t}$ is small. In fact, it causes the high efficiency of the perpendicular recording head in the presence of a keeper layer.

Equation (3) can be rewritten as:

$$
M=-\left(I+\frac{t}{g}\right) H+\frac{n I}{g}
$$

which gives us, apart from the hysteresis loop of fig. 2, a second relation between $M$ and $H$ in the recording layer. It is clear that the presence of the head $(g<\infty)$ causes a steepening of the slope of the initial relation $M=-H$, while a magnetomotive force nI driving the head causes a vertical shift of the line (fig. 2). The initial situation being $A$, the positioning of the head will decrease the magnetic field in the recording layer until $B$ is reached. A magnetonotive force $n I=t . H_{C}$ will restore situation $A$, a further increase of $n I$ causes a change of magnetization, for instance until $\mathrm{C}$ is reached. A decrease of $\mathrm{nI}$ to zero will then result in situation $\mathrm{D}$ and removing the head will cause the magnetization to relax to E.

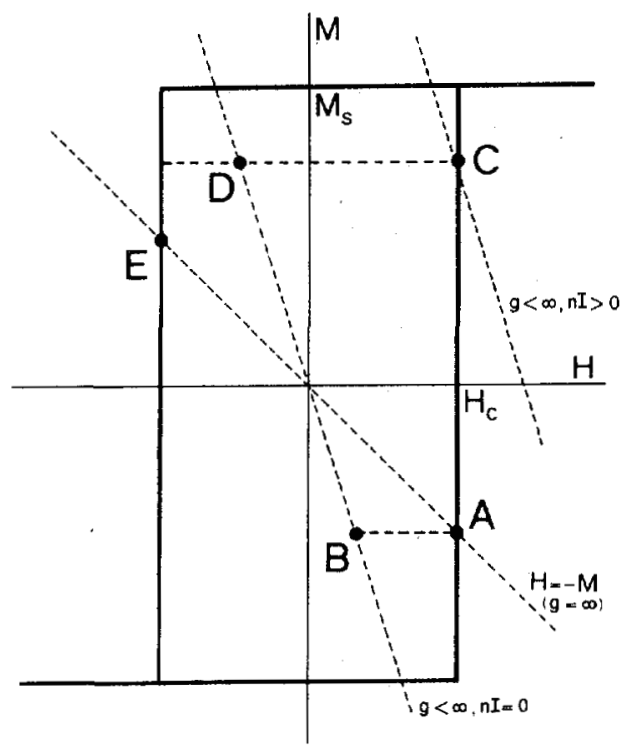

Fig. 2. Assumed intrinsic hysteresis loop of the recording layer.

RECORDING PROCESS NEAR THE EDGE OF THE HEAD

General.

Near the edge of the head, the image-charge field is still significant. Its computation is not trivial, and has so far been neglected in literature. The image-charge influence can be derived from a computation of the magnetization distribution in the edge region, which is based on the assumption that $\mathrm{H}=\mathrm{H}_{C}$ in every part of the recording layer during writing. This is shown by wielinga et al. [4] and was already illustrated by fig. 2 .

The recording layer region near the edge of the head is subdivided into $n$ narrow stripes having magnetic fields $\mathrm{H}_{j}$ 
and magnetizations $M_{j} \quad(1<=j<=n)$. We consider $\mathrm{H}_{1}, \ldots, \mathrm{H}_{\mathrm{n}}$ and $\mathrm{M}_{1}, \ldots \mathrm{M}_{\mathrm{n}}$ as two n-dimensional vectors $\mathbf{B}$ and $M$ respectively. The magnetic field in each stripe consists of three contributions: 1) the local head field $H_{h}, 2$ ) the contribution $H_{f}$ of the recording layer regions outside the subdivided area, where the magnetization is assumed to be constant, and 3) the contribution $h_{m}$, caused by the magnetization in all $n$ considered stripes. The vector $H_{m}$, consisting of the $n$ values of $H_{m}$, can therefore be written as [D].M, where [D] is an $n^{*} n$-matrix containing the magnetic interactions between each pair of stripes. The sum of the field contributions, which as stated must equal $\mathrm{H}_{C}$, can now be written as:

$$
\mathbf{H}_{\mathbf{H}}+\mathbf{H}_{\mathbf{f}}+[\mathrm{D}] \cdot \mathbf{H}=\mathbf{H}_{\mathbf{C}}
$$

where $\mathrm{H}_{c}$ is $a$ vector containing in elements with a numerical value of $\mathrm{H}_{C}$. This implicit solution of $M$ can be made explicit by inverting [D] and rearranging the terms:

$$
M=[D]^{-1}\left\{\mathbf{H}_{C}-\mathbf{H}_{\mathbf{h}}-\mathbf{H}_{\mathrm{f}}\right\}
$$

Now, $M$ can easily be calculated if $[D], H_{h}$ and $\mathbf{H}_{f}$ are known.

Without inage charges taken into account.

If we neglect the image charges, the only influence the magnetization $M_{i}$ in the $i-t h$ stripe performs on the magnetic field $H_{j}$ in the $j$-th stripe is the selfdemagnetization, which can be calculated by using Coulomb's law for magnetic charges:

$$
\overrightarrow{\mathrm{H}}=\int \frac{-(\nabla \cdot \overrightarrow{\mathrm{M}}) \cdot \overrightarrow{\mathrm{r}}}{4 \pi \mathrm{r}^{3}} \mathrm{dV}
$$

A unit surface charge Mdxdz on the top surface and a unit charge $-M d x d z$ on the bottom surface of the recording layer together induce a field $\mathrm{dH}_{\mathrm{y}}$ at a specified point $(x, y, z)$, which can be calculated with (7) (fig. 3). Note that the concentration of magnetic charge in the top and bottom surfaces of the recording layer implies the assumption of a homogeneous magnetization distribution within each of the stripes. Integrating Mdxdz over the top and bottom surface of the i-th stripe $\left(x_{i}<x<x_{i}+\Delta x\right.$ and $\left.-\infty<z<\infty\right)$ and integrating alfy over the face of the $j$-th stripe $\left(x_{j}<\right.$ $x<x_{j}+\Delta x$ and $\left.0<y<t\right)$ gives us the possibility to compute the contribution of the magnetization in the $i-t h$ stripe to the magnetic field in the $j-$ th stripe. Evaluation for aIl combinations of $i$ and $j$ will yield [D]. It is clear that $d_{i j}$ only depends on the distance between the $i-t h$ and $j$-th stripe, so [D] will be a symmetric tensor. $\mathbf{H}_{f}$ can be calculated similarly by considering the recording layer regions outside the subdivided area as "stripes" with an infinite width.

The top surface of the keeper layer is an equipotential surface, just as the symetry-axis of a ring head is (fig. 4). The given geometry can therefore be considered as one half of a ring head, with the recording layer running through, instead of

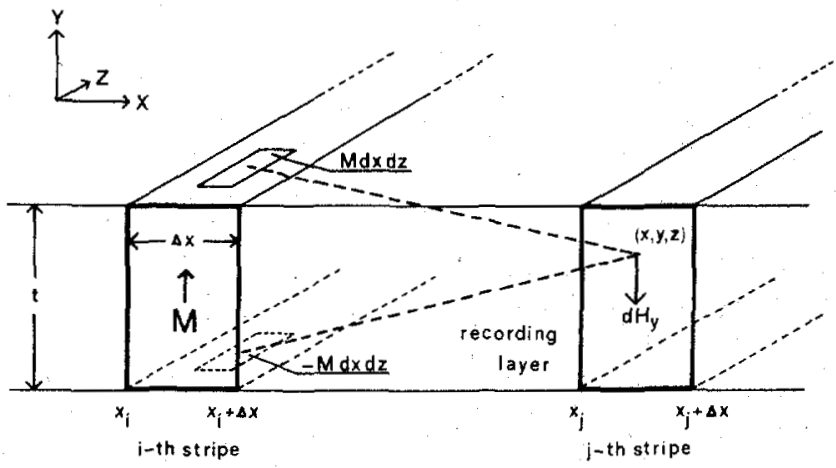

Fig. 3. Determination of $d_{i j}$ without taking the image charges into account. along, the gap. Therefore, $\mathbf{B}_{\mathrm{h}}$ can be approximated by a known analytical function (Karlquist [5], Szczech [6]), but can also be calculated as discussed in the next subsection.

\section{With image charges taken into account.}

For the computation of [D] including the influence of image charges, the contours of the pole pieces of fig. 4B are transformed into a straight line by a conformal mapping method (fig. 4). The top surface of the recording layer will map into a curve in the right half plane and any magnetization on that surface will be mirrored by the above mentioned straight line, Every $d_{i j}$ can be recomputed by transforming the end surfaces of both the $i-t h$ and $j$-th stripes, assuming unity magnetic charges with opposite signs in the end surfaces of the i-th stripe and deriving the potential difference between the end surfaces of the $j$-th stripe in the transformed domain. This potential difference will not be affected by the (inverse) transformation, so that dividing it by the recording layer thickness $t$ will result in $d_{i j}$. Now, $d_{i j}$ will depend on both the distance between the $i-t h$ and $j$-th stripe, as well as their positions with respect to the edge of the head. Therefore, the symmetry of [D] will be disturbed. $\mathbf{H}_{f}$ can again be computed as the effect of infinitely sized "stripes"

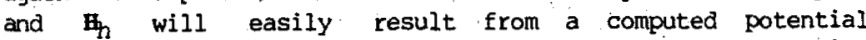
difference in the transformed domain with a known potential difference forced on the transformed parts of the pole pieces of the equivalent ring head.

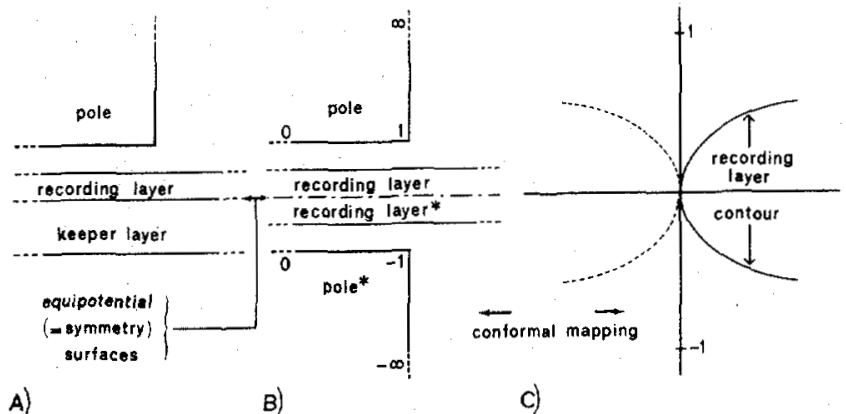

Fig. 4. A) Given pole-keeper head geometry.

B) Equivalent ring head geometry.

C) Resulting geometry after conformal mapping.

\section{RESULTS AND DISCUSSION}

Figure 5 illustrates the significance of the image-charge field. Without it, the magnetization tends to peak near the transition, due to the fact that the recording layer near the transition is magnetized rather than demagnetized by its righthand part. The image-charge field partially compensates the self-demagnetizing field. This can be considered as an enhancement of the effective field underneath the pole as well as a reduction of the self-demagnetization within the transition. The result is the disappearance of the magnetization peak (unless $\mathrm{g} / \mathrm{t}<<1$ ) and an increase of the deepgap magnetization. It is clear that the smaller $\mathrm{g} / \mathrm{t}$ is chosen, the more influence the image-charge field will have. It was observed that with $g / t>>1$ (an unpractical situation), the magnetization distributions computed with and without the image charges, although computed by essentially different algorithms, were exactly the same. Figures 5 and 6 illustrate the effect for two $g / t$-ratios.

After computing a transition with image charges taken into account, the self-demagnetizing field that is caused by the computed magnetization distribution can be calculated with Coulomb's law as mentioned previously. The contribution of the image-charge field can now be calculated by subtracting both this self-demagnetizing field as well as the head field from $H_{c}$. The result of this split is shown in fig. 7. Adding the image-charge field to the head field results in a higher effective head field and head-field gradient, causing a higher and steeper transition, as already shown (fig. 5). The gradient of the image-charge field itself however, is smaller 
than that of the head field. Normalization on the deep-gap magnetization therefore, results in a smaller gradient of magnetization ( $\mathrm{fig} .8$ ).

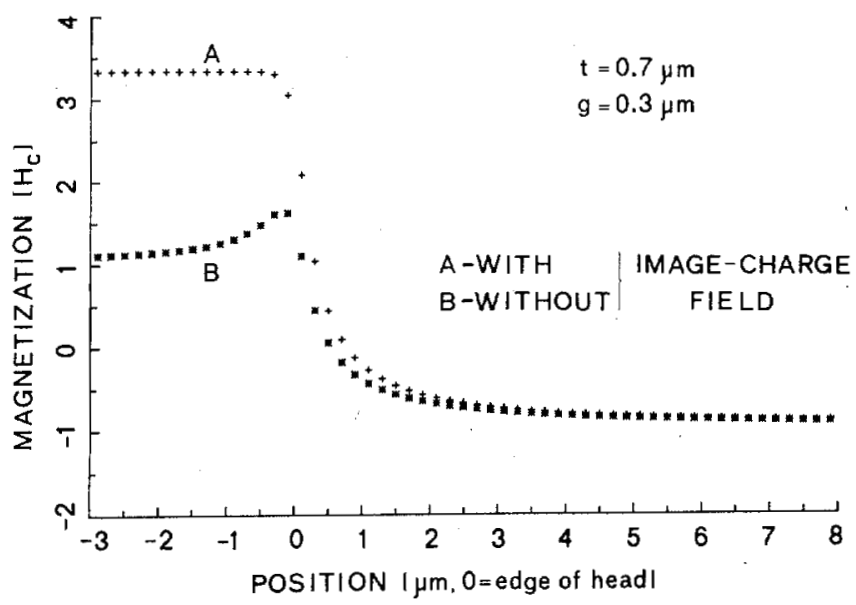

Fig. 5. Magnetization distribution with and without the image-charge field taken into account $(t=0.7 \mu \mathrm{m}$, $g=0.3 \mu \mathrm{m})$. The deep-gap head field equals $2 \mathrm{H}_{\mathrm{C}}$ in both cases.

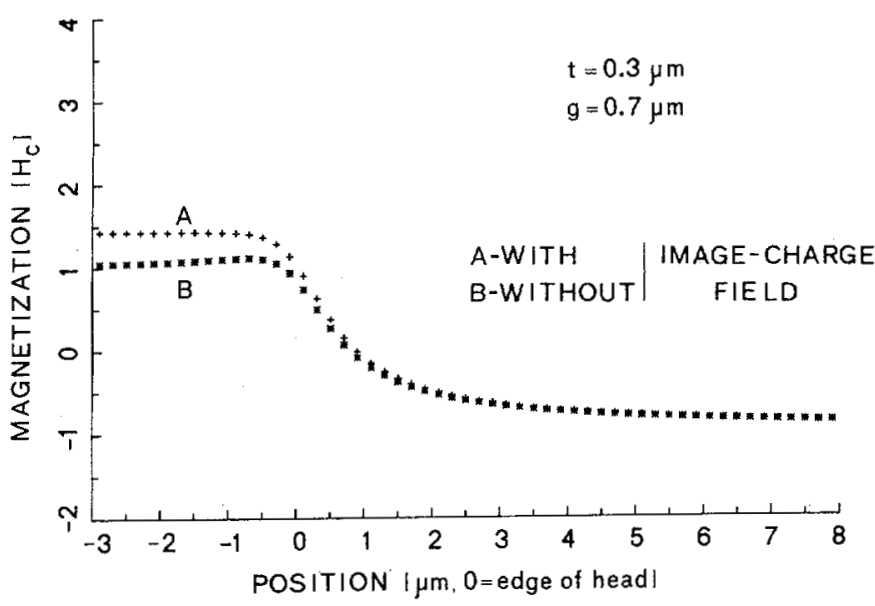

Fig. 6. Magnetization distribution with and without the image-charge field taken into account ( $t=0.3 \mu \mathrm{m}$, $\mathrm{g}=0.7 \mu \mathrm{m})$. The deep-gap head field equals $2 \mathrm{H}_{C}$ in both cases.

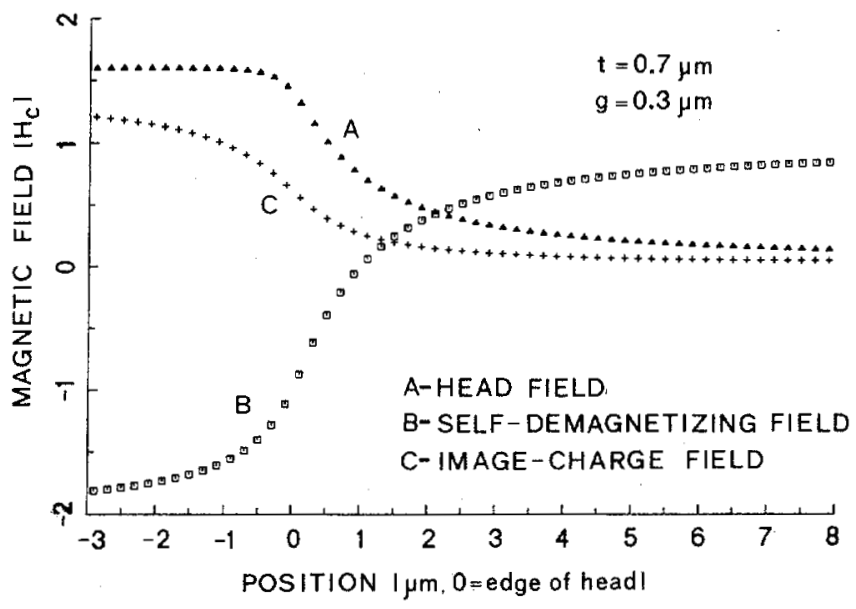

Fig. 7. Contribution of the head field, the self-demagnetizing field and the image-charge field at a deep-gap head field of $1.6 \mathrm{H}_{\mathrm{c}}(\mathrm{t}=0.7 \mu \mathrm{m}, \mathrm{g}=0.3 \mu \mathrm{m})$.

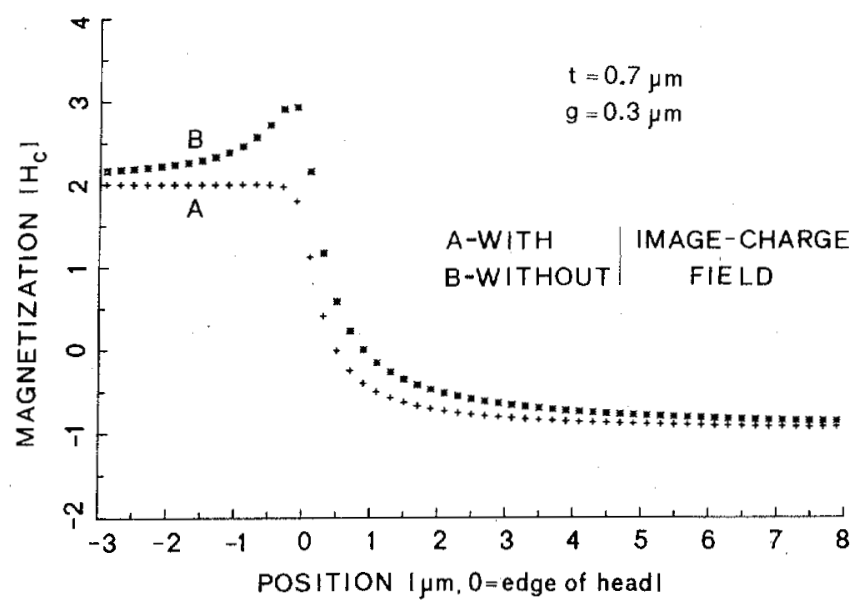

Fig. 8. Magnetization distribution with and without the image-charge field taken into account $(t=0.7 \mu \mathrm{m}$, $g=0.3 \mu \mathrm{m})$. The deep-gap head fields are such, that the deep-gap magnetization equals $2 \mathrm{H}_{\mathrm{C}}$ in both cases.

The results are given with the activated head in position. The removal of the head will cause the total field to decrease. Under the given assumption, a rectangular intrinsic hysteresis loop with rectangular minor loops, all magnetization values will remain constant under the decreasing field until the lefthand edge of the hysteresis loop is reached. The relaxation process which will then take place, can also be described by an algorithm. It is clear that the magnetization of the part of the recording layer which is in the deep-gap region during writing, will ultimately relax to $M=\mathrm{H}_{C}$. This will cause the typical self-magnetization peak in the transition region to ocur, as was already observed in a preliminary run of the relaxation algorithm. Results will be published in the near future.

\section{CONCLUSIONS}

It can be concluded from the computed magnetization and magnetic-field distributions of a transition in a perpendicular recording layer that the effects of image charges are significant and geometry-dependent. Their influence can be considered as an enhancement of the magnetomotive force by which the head is driven, resulting in higher and steeper transitions. On the other hand, the effect on the height of the transition is larger than that on the gradient of magnetization. Therefore, if the magnetization under the head is normalized, a lower maximum gradient in the transition is found. The self-magnetization peak, a typical feature of a perpendicularly recorded transition, is not present during writing, but will appear after the head is removed from the recording layer.

\section{REFERENCES}

1) S. Iwasaki, Y. Nakamura, S. Yamamoto, K. Yamakawa. "Perpendicular Recording by a Narrow Track Single Pole Head". I.E.E.E. Trans. on Magn., Vol. MAG-19, No. 5, pp. 1713-5, September 1983.

2) J.C. Mallinson, H.N. Bertram. "On the Characteristics of pole-Keeper Head Fields". I.E.E.E. Trans. on Magn., Vol. MAG-20, No. 5, pp. 721-3, September 1984.

3) A. Ohtsubo, y. Satoh. "Thick Non-Parallel Single Pole Head for Perpendicular Recording from One Side". I.E.E.E. Trans. on Magn., Vol. MAG-18, No. 6, pp. 1173-5, November 1982.

4) T. Wielinga, J.H.J. Fluitman, J.C. Lodder. "Perpendicular Stand-Still Recording in CoCr-Films". I.E.E.E. Trans. on Magn., Vol. MAG-19, No. 2, pp. 94-104, March 1983.

5) 0. Karlqvist. "Caiculation of the Magnetic Field in the Ferromagnetic Layer of a Magnetic Drum". Trans. Royal Inst. of Techn. Stockholm, Vol. 86, pp. 1-27, 1954.

6) T.J. Szczech, D.M. Perry, K.E. Palmqvist. "Improved Field Equations for Ring Heads". I.E.E.E. Trans. on Magn., Vol. MAG-19, No. 5, pp. 1740-4, September. 1983. 